\title{
Commentary: Neurotransmitter Secretion and Cell Signaling
}

\author{
Lung-Sen Kao $・$ Y. Peng Loh
}

Published online: 17 May 2012

(C) Springer Science+Business Media, LLC (outside the USA) 2012

Many proteins involved in neurotransmitter release have been identified, but how these molecules interact and function together to mediate neurotransmission is not completely known. Chromaffin cells have been one of the favorite models for the study of the molecular mechanism of exocytosis and regulation of the differential release of catecholamine and peptides co-stored in the chromaffin granules. Several talks were presented at this meeting, describing the function of different molecules in regulating neurotransmitter secretion and synaptic transmission.

Corey Smith (USA) reported that by using various frequencies to mimic basal and acute sympathetic activity to stimulate chromaffin cells, he was able to demonstrate that differential release of neurotransmitters is regulated by the opening of the fusion pore mediated by an interaction between syndapin and dynamin. Xuelin Lou (USA) presented new data on the role of dynamin isoforms in synaptic transmission by showing that presynaptic dynamin 3 function synergizes with dynamin 1. Significantly, neurons lacking both dynamins develop, differentiate, and establish synapses in vitro. But most strikingly, nerve terminals can recycle synaptic vesicles in the absence of both dynamins, implying that dynamin 2 alone and/or dynamin independent mechanisms are sufficient to support basic synaptic function. These results collectively

\section{L.-S. Kao}

Department of Life Sciences and Institute of Genome Sciences, Brain Research Center, National Yang-Ming University,

Taipei, Taiwan

e-mail: 1skao@ym.edu.tw

\section{Y. P. Loh $(\bowtie)$}

Section on Cellular Neurobiology, Program on Developmental Neuroscience, Eunice Kennedy Shriver National Institute of Child Health and Human Development, National Institutes of Health, Bethesda, MD 20892, USA

e-mail: lohp@mail.nih.gov demonstrate that neither dynamin 1 nor 3 are essential for regenerating synaptic vesicles, but rather contribute to the efficiency of this process. Chen Zhang (China) discussed the role of presenilin, a molecule implicated in the pathogenesis of Alzheimer's disease, in neurotransmitter secretion. He used genetic approaches to conditionally inactivate the protein in presynaptic and postsynaptic neurons in the hippocampus. His results showed that only presynaptic, but not postsynaptic inactivation of presenilin impaired synaptic activity and this impairment was induced by modulation of $\mathrm{Ca}^{2+}$ release from the endoplasmic reticulum $\mathrm{Ca}^{2+}$ stores. Kathrin Engisch (USA) used the isolated mouse chromaffin cells to study the effects of levetiracetam, an anticonvulsant that binds to the synaptic vesicle protein SV2A on neurotransmitter release. She showed that levetiracetam decreases the high activity-induced $\mathrm{Ca}^{2+}$ currents and secretion but had no effect on the resting cells. The results may shed some light on the pharmacology of the drug on neurotransmission. The effects of some molecules on catecholamine secretion were also presented. Damien Keating (Australia) discussed interesting results on the molecular mechanism of exocytosis regulated by HAP1, Huntingtin-associated protein 1. Kevin Curie (USA) reported the paracrine effects of $\mathrm{PGE}_{2}$ on the secretion, possibly mediated by EP3 receptor. Daniel O'Connor (USA) reported a systematic study of the polymorphism found in the enzymes involved in catecholamine biosynthesis.

G-protein coupled receptors (GPCR) and glutamate receptors play major roles in cell signaling. Duk-Su Koh (USA) presented studies on glutaminergic signaling in pancreatic islets. Extending his presentation at the meeting, he has provided a comprehensive review (below) on how islet hormones such as insulin and glucagon act as paracrine or autocrine modulators of neighboring cells in the pancreatic islets to regulate glucose levels. In addition, he has described a role of excitatory glutamate and inhibitory $\gamma$-amino butyric 
acid (GABA) neurotransmitters that are co-released with hormones, in activating or inhibiting cells within the islets. In this section several papers relating to cell signaling through glutamate receptors contributed by attendees of the meeting are also included. Chen Zhang in a paper below reviewed the interacting partners of AMPA-type glutamate receptors. $\mathrm{Ji}$ Min Cao and Zhuan Zhou present their work (paper below) on the upregulation of glutamate receptors during nerve sprouting after myocardial necrotic injury in rats which increases the severity of ventricular tachyarrhythmia. Xiao $Y u$ presented a poster and describes his studies in a paper (below), on the effects of activation of cholescystokinin (CCK) A receptor, a GPCR, by CCK, on insulin secretion in pancreatic $\beta$-cells. Pei-Shan Liu and Yi-Yun Kao (Taiwan) presented a poster, and in a paper below they further describe the effect of translocation of $\mathrm{ZnO}$ nanoparticles into $\mathrm{PC} 12$ cells on alteration of nicotinic receptor expression.

All the papers presented in this section using chromaffin cells and pancreatic islet cells illustrate the complexity of autocrine/paracrine cell signaling in the regulation of cellular and higher physiological function through specific neurotransmitter and hormone release, acting via their respective receptors. 